\title{
A Partial Response of Pulmonary Pleomorphic Carcinoma to Camrelizumab (PDI Monoclonal Antibody) Monotherapy: A Case Report
}

This article was published in the following Dove Press journal: OncoTargets and Therapy

\author{
Yi Chen' \\ Hao Zhang ${ }^{2}$ \\ Junfeng $\mathrm{Shi}^{3, *}$ \\ Tongshan Wang ${ }^{2, *}$ \\ 'Department of Oncology, Nanjing \\ Pukou Central Hospital, Pukou Branch \\ Hospital of Jiangsu Province Hospital, \\ Nanjing 210000, People's Republic of \\ China; ${ }^{2}$ Department of Oncology, Jiangsu \\ Provincial People's Hospital, First \\ Affiliated Hospital of Nanjing Medical \\ University, Nanjing 210000, People's \\ Republic of China; ${ }^{3}$ Department of \\ Oncology, Nanjing First Hospital, Nanjing \\ Medical University, Nanjing 210000, \\ People's Republic of China \\ *These authors contributed equally to \\ this work
}

\begin{abstract}
We report a case of a 68-year-old man diagnosed with pulmonary pleomorphic carcinoma who showed partial response after a single treatment with camrelizumab (PD1 monoclonal antibody). The patient's tumor was positive for programmed cell death ligand 1 (PD-L1) and progressed rapidly after a course of chemotherapy. Fortunately, the tumors dramatically shrank after one cycle of camrelizumab, an anti-programmed cell death-1 (PD1) antibody developed by Chinese Hengrui Medicine. In conclusion, camrelizumab may be a good treatment option, especially in tumors that express PD-L1.
\end{abstract}

Keywords: camrelizumab, pulmonary pleomorphic carcinoma, immune checkpoint inhibitor, PD-1, reactive skin capillary endothelial cell proliferation, RCCEP

\section{Introduction}

Pulmonary pleomorphic carcinoma (PPC) is defined as poorly differentiated nonsmall cell lung cancer (NSCLC) with its incidence ranging from $0.1 \%$ to $0.4 \%$ of all lung cancer. ${ }^{1}$ It is characterized by a cancer that contains at least $10 \%$ spindleshaped and/or giant cells, or consists only of spindle-shaped and/or giant cells. ${ }^{2}$ Although surgical resection in early patients sometimes produces good results, ${ }^{3,4}$ PPC usually produces disappointing clinical results due to resistance to conventional chemotherapy and lack of specific targeted drug treatment. ${ }^{1,3-5}$

PPC has been recently reported with a high expression level of programmed death ligand 1 (PD-L1). ${ }^{6}$ Certain cases have shown successful treatment with immune checkpoint inhibitors (ICIs) such as pembrolizumab and nivolumab, ${ }^{7-10}$ which have been approved for the treatment of non-small cell lung cancer. ${ }^{9,11}$ Camrelizumab (PD1 monoclonal antibody; AiRuiKa ${ }^{\mathrm{TM}}$ ) is a programmed cell death 1 (PD-1) inhibitor developed by Jiangsu Hengrui Medicine Co., Ltd. It has received conditional approval in China for the treatment of recurrent or refractory classic Hodgkin Lymphoma1, ${ }^{12}$ but its efficacy in PPC is currently unknown.

Here, we report that a rare stage IV inoperable PPC patient showed high PD-L1 expression, and this patient experienced a rapid partial response after receiving only camrelizumab.

\section{Case Report}

A non-smoking 68-year-old man came to our hospital in November 2019 for back numbness and chest pain. He underwent partial gastrectomy for gastric malignancy at 
the age of 63. Enhanced computed tomography (CT) showed that the pulmonary mass was a single $5.1 * 5.8 \mathrm{~cm}$, invading the first and second left ribs of the upper left lobe (Figure 1).

Multiple enlargement of lymph nodes in mediastinum and bilateral hilum, axilla, abdominal aorta and mesenteric region was observed. There was no metastasis on brain magnetic resonance imaging. The clinical stage is T3N3M1c stage IVB (the eighth TNM classification of lung cancer). Histological examination of CT-guided percutaneous lung biopsy specimens from the left lung mass showed lung polymorphic carcinoma (Figure 2A). No epidermal growth factor receptor (EGFR) gene mutation and anaplastic lymphoma kinase gene were detected. PD-L1 tumor proportion score (TPS) $>90 \%$ (Dako 22C3 IHC platform) detected by Burning Rock Dx (Figure 2B, Supplementary Materials).

After one course of chemotherapy with cisplatin and paclitaxel, the patient had significant vomiting and was diagnosed with intestinal obstruction by CT scan. The symptoms are relieved by symptomatic treatment. However, after 21 days of the administration, chest CT images revealed the enlargement of the tumor with $6.3 * 7.2 \mathrm{~cm}$ in diameter (Figure 3). Considering adverse events and adverse effects, we recommend the use of PD-1 inhibitors, such as nivolumab or pembrolizumab. Due to financial deficiencies, the patient refused to accept the drug and received second-line treatment with camrelizumab (200 mg every 2 weeks) in December 2019. It was found that the left upper lobe tumor had been significantly reduced after only two weeks of treatment with camrelizumab. After another 2 treatment cycles, cancerous changes were seen on CT (Figure 4A and B). After five cycles of $\mathrm{CT}$, a partial response was confirmed (solid tumor response evaluation criteria, version 1.1) (Figure 4C).
A

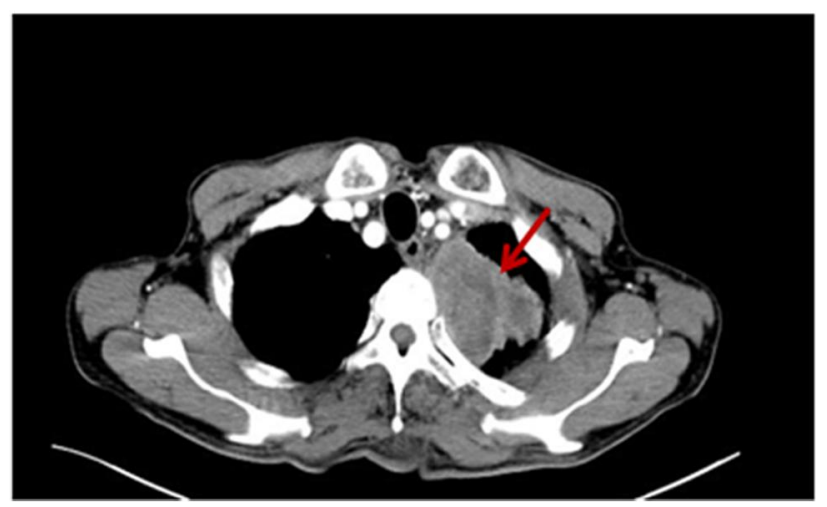

B

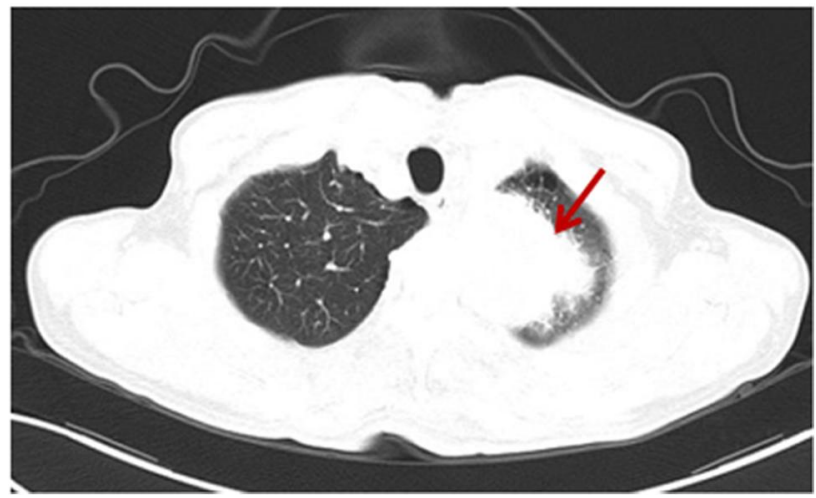

Figure I Computed tomography at the first presentation showing a 5 -cm massive pulmonary tumor in the left upper lobe and multiple nodules in both lung fields. (A) Mediastinal window, (B) pulmonary window.

A

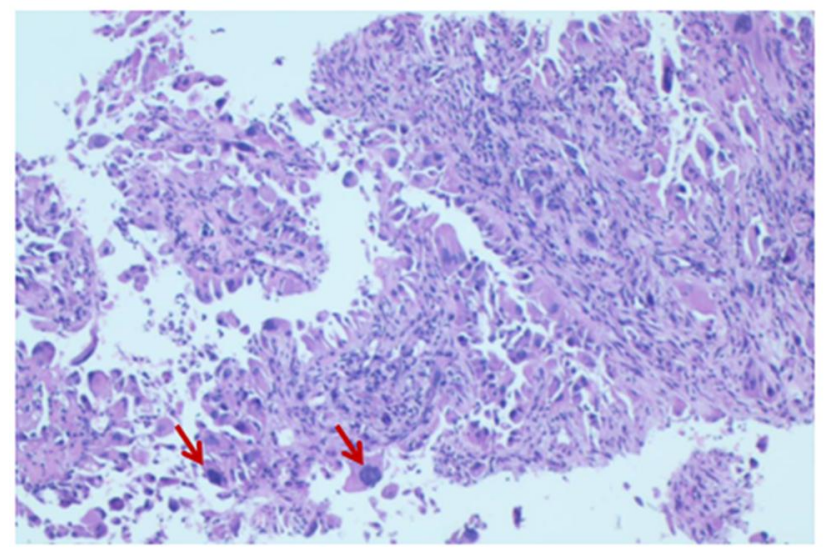

B

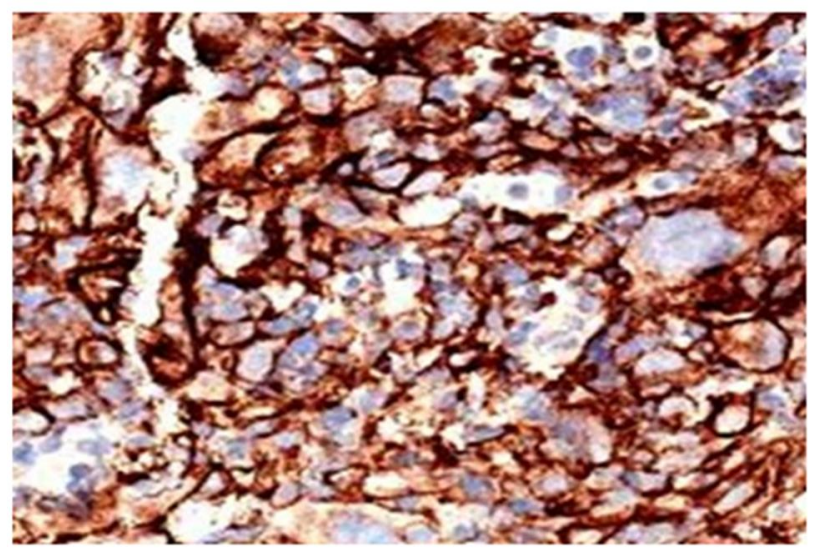

Figure 2 (A) Histological examination of lung biopsy specimens obtained from the left pulmonary mass revealing proliferation of polyhedral and spindle atypical cells. (B) PD-LI tumor proportion score was detected by Burning Rock Dx. 


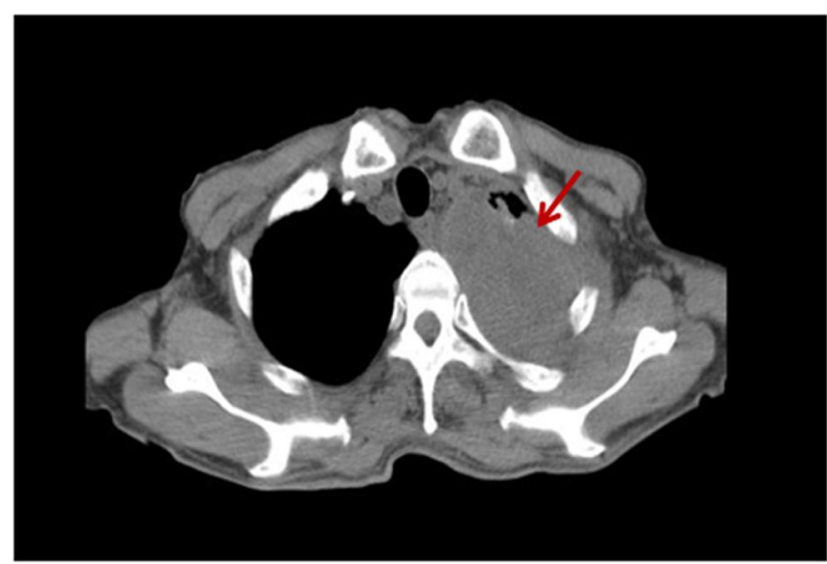

Figure 3 Chest CT images obtained after one course of chemotherapy.

During the course of treatment, except for reactive skin capillary endothelial cell proliferation (RCCEP), no serious adverse events were observed (Figure 5A). Since RCCEP is most located on the scalp and trunk was given, a small molecule intravascular angiogenesis factor 2 receptor 2 (VEGFR-2) tyrosine kinase inhibitor, and RCCEP was significantly reduced (Figure 5A and B). At the same time, the expression of VEGFR2 in tumor tissues was significantly decreased (Figure 6). At the time of writing, camrelizumab treatment has been maintained for 9 cycles, with a tumor diameter of $2.6 * 1.4 \mathrm{~cm}$.

\section{Discussion}

PPC has a worse outcome than other NSCLC. Fishback et al firstly proposed the conception of pulmonary pleomorphic carcinoma in $1994 .{ }^{3}$ The average age of patients with pleomorphic carcinoma was about 60 years old, and it was more common in males than females, with a male to female ratio 5:1, which was closely related to smoking. ${ }^{1,3,13}$ In general, the resistance of patients with PPC to platinum and non-platinum cytotoxic chemotherapy and radiation therapy shows an aggressive clinical
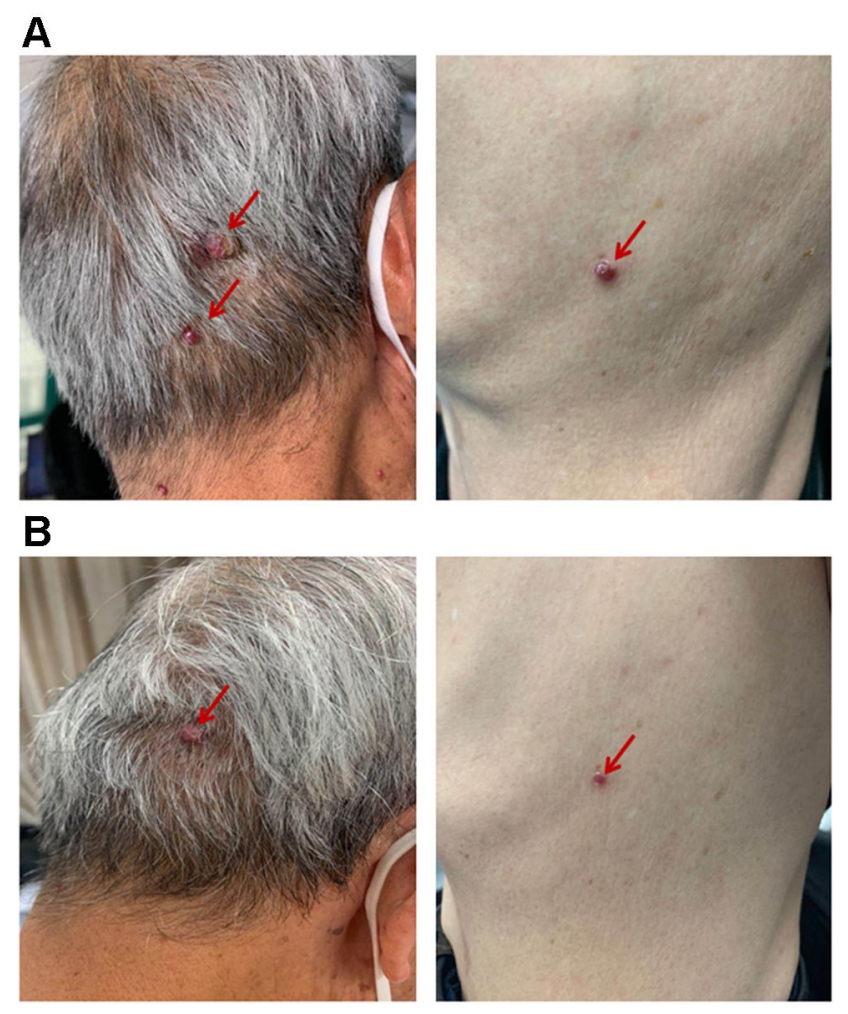

Figure 5 Reactive cutaneous capillary endothelial cell proliferation (RCCEP) was located on the scalp and trunk of patients before (A) and after (B) apatinib treatment.

course and poor prognostic outcomes. ${ }^{1,3-5}$ Nakanishi et al reported that 13 of 22 patients who received surgical resections relapsed within a few months. ${ }^{7}$ Bae et al reported cytotoxic chemotherapy was administered for postoperative relapse and inoperable cases and 11 of 13 cases involved progressive disease, with a median overall survival (OS) of approximately five months. ${ }^{4}$ Similarly, it has been reported that some PPC cases receiving cytotoxic chemotherapy have a median OS of only 7-8 months. ${ }^{14}$

Recently, some studies reported that immunity checkpoint inhibitors (ICI) for cases with high expression of PD-L1 in
A

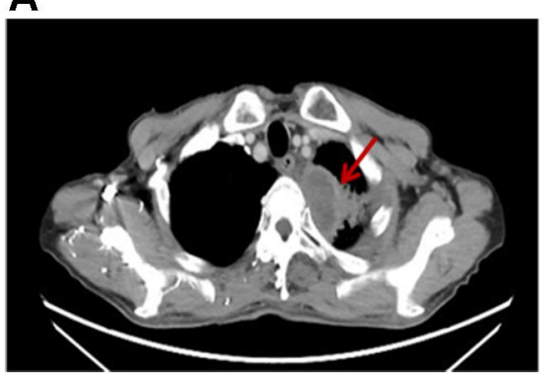

B

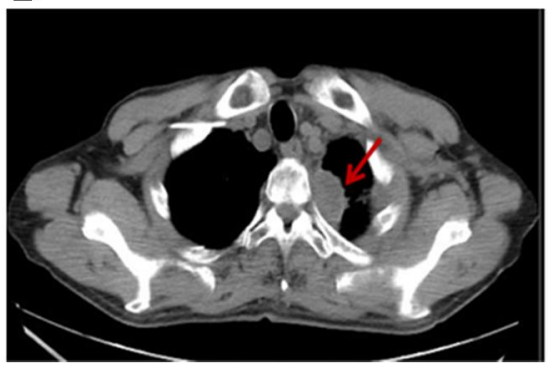

C

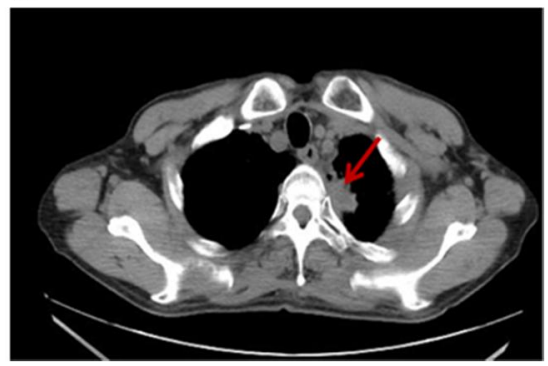

Figure 4 Chest CT images obtained after 2 weeks (A), 6 weeks (B), and 10 weeks (C) in a patient who received single camrelizumab (PDI monoclonal antibody) treatment. 

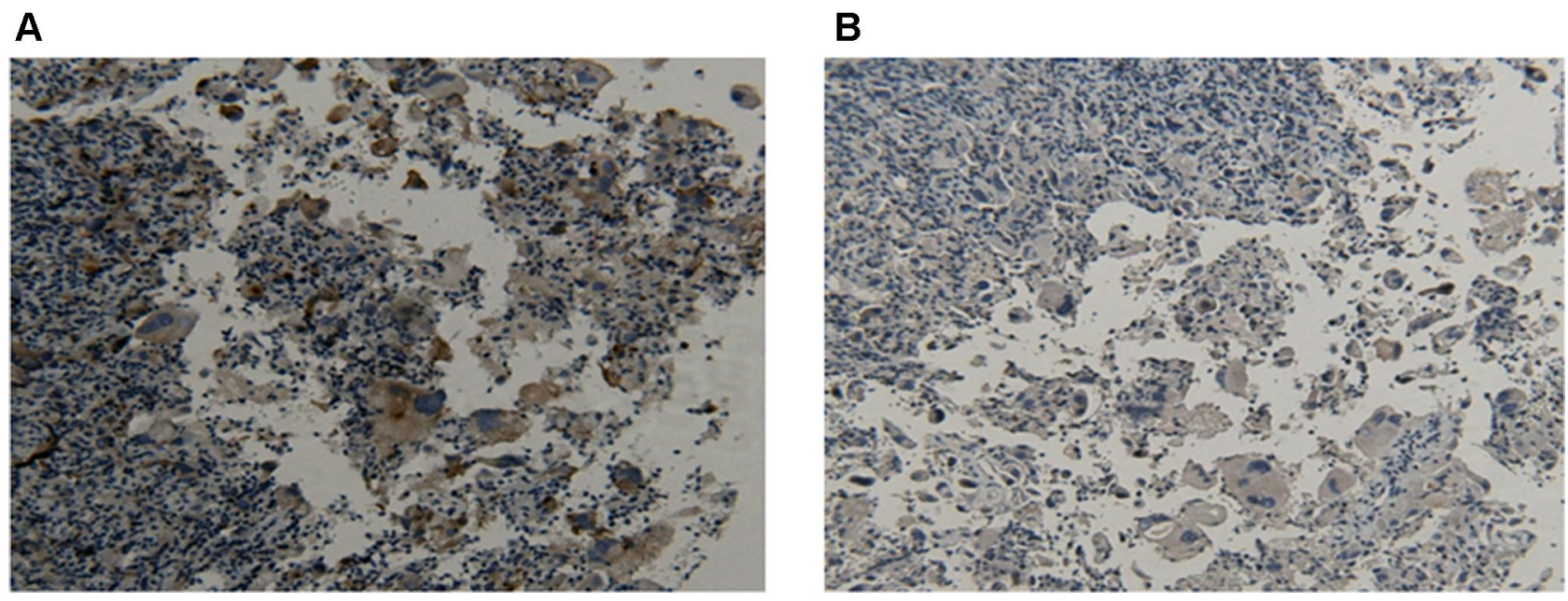

Figure 6 The expression of EGFR2 was detected by IHC before (A) and after (B) apatinib treatment. (I00x).

PPC had improved the treatment outcome. ${ }^{8-11}$ Nivolumab and pembrolizumab are anti-PD-1 monoclonal antibodies, which are most commonly used in clinic. KEYNOTE-021 study showed clinical superiority of pembrolizumab as firstline therapy for advanced NSCLC with chemotherapy. ${ }^{15}$ In another III trial study, nivolumab treatment has been associated with longer progression-free survival and overall survival in comparison to docetaxel in patients with nonsquamous non small cell lung cancer. ${ }^{16}$ Matsumoto et al report a case with PPC obtained durable response after single pembrolizumab treatment. ${ }^{8}$ Some reporters believed that Nivolumab also shows good therapeutic effects in PPC. ${ }^{9}$ However, there are few reports of PD-1 inhibitors other than nivolumab and pembrolizumab in PPC. ${ }^{9,10}$ In this case, we can see that the tumor shrinks rapidly after carlezumab treatment, without obvious adverse effects.

Camrelizumab, a PD-1 inhibitor developed by Jiangsu Hengrui Pharmaceutical Co., Ltd., China. The drug is also being investigated as a treatment for various other malignancies, including B cell lymphoma, ${ }^{17}$ oesophageal squamous cell carcinoma, ${ }^{18}$ gastric/gastroesophageal junction cancer, ${ }^{19}$ hepatocellular carcinoma, ${ }^{20}$ nasopharyngeal cancer $^{21}$ and nonsquamous, non-small cell lung cancer. ${ }^{22} \mathrm{~A}$ case report showed complete response of early-stage hepatocellular carcinoma in a patient treated with combination therapy of camrelizumab and apatinib. ${ }^{23}$ A randomized Phase 3 study demonstrated that first-line camrelizumab plus chemotherapy shows substantial clinical benefit in patients with advanced/metastatic nonsquamous NSCLC with negative EGFR or ALK in terms of progression-free survival (PFS), objective response rate (ORR), and OS and acceptable safety profiles. ${ }^{24}$
The IC50 and EC50 of camrelizumab are similar to Papolizumab, far lower than other PD-1 monoclonal antibodies camrelizumab has a high affinity for PD-1 receptors and a high occupancy rate for PD-1 receptors, which can effectively block the PD-1/PD-L1 pathway, activate T cells and promote the release of IFN in T cells. ${ }^{25}$ In animal models, camrelizumab has been shown to have stronger tumor-suppressive effects than other similar drugs, and has been shown to be effective against different types of relapsed/refractory tumors, such as classic Hodgkin lymphoma, relapse/metastasis Nasopharyngeal carcinoma and advanced liver cancer.

In terms of safety, the overall incidence of camrelizumab and immune-related adverse reactions is very low. More than 900 patients participating in the clinical trial of camrelizumab, in addition to RCCEP, the incidence of immune-related adverse reactions is often lower than similar products. ${ }^{26,27}$ This may be related to the short half-life of camrelizumab.

Multiple studies revealed that PPC with high PD-L1 expression levels responds remarkably well to PD-1 inhibitors, which may imply that the expression of PD-L1 in PPC is an indication for anti PD-1 antibody treatment. More detailed mechanisms need to be explored, and it is necessary to further study the use of camrelizumab in patients with PPC.

\section{Conclusion}

To the best of our knowledge, this is the first report of a significant reaction to PD-L1 positive PPC patients to camrelizumab. We prove that camrelizumab seems to provide effective and easy treatment for PPC with high 
expression levels of PD-L1, and can significantly improve disease burden, quality of life and survival time.

\section{Ethics and Consent Statement}

The patient agreed and submitted a written informed consent to allow publication of the details of the case. An institutional approval was not required for a case report.

\section{Acknowledgments}

This study was supported by Natural Science Foundation of Jiangsu Provincial Department of Education (17KJB320007), CSCO-Hausoh Cancer Research Foundation (Y-HS2017-032), the Health Science and Technology Development Foundation of Nanjing (Grant No.JQX18004), the project of Science \& Technology of Nanjing (YKK16248).

\section{Disclosure}

The authors declare that they have no conflicts of interest.

\section{References}

1. Chang YL, Lee YC, Shih JY, Wu CT. Pulmonary pleomorphic (spindle) cell carcinoma: peculiar clinicopathologic manifestations different from ordinary non-small cell carcinoma. Lung Cancer 2001;34:91-97. doi:10.1016/S0169-5002(01)00224-0

2. Travis WD. Sarcomatoid neoplasms of the lung and pleura. Arch Pathol Lab Med. 2010;134:1645-1658.

3. Fishback NF, Travis WD, Moran CA, Guinee DG Jr, McCarthy WF, Koss MN. Pleomorphic (spindle/giant cell) carcinoma of the lung. A clinicopathologic correlation of 78 cases. Cancer. 1994;73:2936-2945. doi:10.1002/1097-0142(19940615)73:12<2936:: AID-CNCR2820731210>3.0.CO;2-U

4. Bae HM, Min HS, Lee SH, et al. Palliative chemotherapy for pulmonary pleomorphic carcinoma. Lung Cancer. 2007;58:112-115. doi:10.1016/j.lungcan.2007.05.006

5. Ito K, Oizumi S, Fukumoto S, et al. Clinical characteristics of pleomorphic carcinoma of the lung. Lung Cancer. 2010;68: 204-210. doi:10.1016/j.lungcan.2009.06.002

6. Kim S, Kim MY, Koh J, et al. Programmed death-1 ligand 1 and 2 are highly expressed in pleomorphic carcinomas of the lung: comparison of sarcomatous and carcinomatous areas. Eur J Cancer. 2015; 51:2698-2707. doi:10.1016/j.ejca.2015.08.013

7. Nakanishi K, Sakakura N, Matsui T, et al. Clinicopathological features, surgical outcomes, oncogenic status and PD-L1 expression of pulmonary pleomorphic carcinoma. Anticancer Res. 2019;39:57 89-5795. doi:10.21873/anticanres. 13782

8. Matsumoto Y, Miura T, Horiuchi H, Usui K. The successful treatment of pulmonary pleomorphic carcinoma with pembrolizumab: a case report. Case Rep Oncol. 2017;10:752-757. doi:10.1159/000479552

9. Senoo S, Ninomiya T, Makimoto G, et al. Rapid and long-term response of pulmonary pleomorphic carcinoma to nivolumab. Intern Med. 2019;58:985-989. doi:10.2169/internalmedicine.0890-18

10. Ikematsu Y, Yoneshima Y, Ijichi K, et al. Marked response to pembrolizumab in a patient with pulmonary pleomorphic carcinoma highly positive for PD-L1. Lung Cancer. 2017;112:230-231. doi:10.1016/j.lungcan.2017.07.020
11. Yaguchi D, Ichikawa M, Ito M, Okamoto S, Kimura H, Watanabe K. Dramatic response to nivolumab after local radiotherapy in pulmonary pleomorphic carcinoma with rapid progressive post-surgical recurrence. Thorac Cancer. 2019;10:1263-1266. doi:10.1111/17597714.13029

12. Markham A, Keam SJ. Correction to: camrelizumab: first global approval. Drugs. 2019;79:1497. doi:10.1007/s40265-01901191-0

13. Weissferdt A, Kalhor N, Rodriguez Canales J, et al. Spindle cell and pleomorphic ("sarcomatoid") carcinomas of the lung: an immunohistochemical analysis of 86 cases. Hum Pathol. 2017;59:1-9. doi:10.1016/j.humpath.2016.08.003

14. Tamura Y, Fujiwara Y, Yamamoto N, et al. Retrospective analysis of the efficacy of chemotherapy and molecular targeted therapy for advanced pulmonary pleomorphic carcinoma. BMC Res Notes. 2015;8:800. doi:10.1186/s13104-015-1762-z

15. Gadgeel SM, Stevenson JP, Langer CJ, et al. Pembrolizumab and platinum-based chemotherapy as first-line therapy for advanced non-small-cell lung cancer: Phase 1 cohorts from the KEYNOTE-021 study. Lung Cancer. 2018;125:273-281. doi:10.10 16/S1470-2045(20)30110-8

16. Reck M, Brahmer J, Bennett B, et al. Evaluation of health-related quality of life and symptoms in patients with advanced non-squamous non-small cell lung cancer treated with nivolumab or docetaxel in CheckMate 057. Eur $J$ Cancer. 2018;102:23-30. doi:10.1016/j.ejca.2018.05.005

17. Mei Q, Zhang W, Liu Y, et al. Camrelizumab plus gemcitabine, vinorelbine, and pegylated liposomal doxorubicin in relapsed/refractory primary mediastinal B-cell lymphoma: a single-arm, open-label, Phase II trial. Clin Cancer Res. 2020;26(17):4521-4530. doi:10.1158/ 1078-0432.CCR-20-0514

18. Huang J, Xu J, Chen Y, et al. Camrelizumab versus investigator's choice of chemotherapy as second-line therapy for advanced or metastatic oesophageal squamous cell carcinoma (ESCORT): a multicentre, randomised, open-label, phase 3 study. Lancet Oncol. 2020;21(6):832-842.

19. Xu J, Zhang Y, Jia R, et al. Anti-PD-1 antibody SHR-1210 combined with apatinib for advanced hepatocellular carcinoma, gastric, or esophagogastric junction cancer: an open-label, dose escalation and expansion study. Clin Cancer Res. 2019;25(2):515-523. doi:10.11 58/1078-0432.CCR-18-2484

20. Qin S, Ren Z, Meng Z, et al. Camrelizumab in patients with previously treated advanced hepatocellular carcinoma: a multicentre, open-label, parallel-group, randomised, Phase 2 trial. Lancet Oncol. 2020;21(4):571-580. doi:10.1016/S1470-2045 (20)30011-5

21. Fang W, Yang Y, Ma Y, et al. Camrelizumab (SHR-1210) alone or in combination with gemcitabine plus cisplatin for nasopharyngeal carcinoma: results from two single-arm, phase 1 trials. Lancet Oncol. 2018;19(10):1338-1350. doi:10.1016/S1470-2045(18)30495-9

22. Wei Z, Yang X, Ye X, et al. Camrelizumab combined with microwave ablation improves the objective response rate in advanced non-small cell lung cancer. $J$ Cancer Res Ther. 2019;15 (7):1629-1634. doi:10.4103/jcrt.JCRT_990_19

23. Zhang Z, Zhou Y, Hu K, et al. Complete response of early stage hepatocellular carcinoma in a patient treated with combination therapy of camrelizumab (SHR-1210) and apatinib. Dig Liver Dis. 2019;51(10):1488-1490. doi:10.1016/j.dld.2019.07.005

24. Zhou C, Chen G, Huang Y, et al. OA04.03 A randomized Phase 3 study of camrelizumab plus chemotherapy as 1st line therapy for advanced/metastatic non-squamous non-small cell lung cancer. J Thorac Cancer. 2019;14:S215-S216. doi:10.1016/j.jtho.2019.08.425

25. Zhao S, Ren S, Jiang T, et al. Low-dose apatinib optimizes tumor microenvironment and potentiates antitumor effect of PD-1/PD-L1 blockade in lung cancer. Cancer Immunol Res. 2019;7(4):630-643. 
26. Wang F, Qin S, Sun X, et al. Reactive cutaneous capillary endothelial proliferation in advanced hepatocellular carcinoma patients treated with camrelizumab: data derived from a multicenter phase 2 trial. J Hematol Oncol. 2020;13(1):47. doi:10.1186/s13045-02000886-2
27. Lv JW, Li JY, Luo LN, et al. Comparative safety and efficacy of anti-PD-1 monotherapy, chemotherapy alone, and their combination therapy in advanced nasopharyngeal carcinoma: findings from recent advances in landmark trials. J Immunother Cancer. 2019;7(1):159. doi:10.1186/s40425-019-0636-7

\section{Publish your work in this journal}

OncoTargets and Therapy is an international, peer-reviewed, open access journal focusing on the pathological basis of all cancers, potential targets for therapy and treatment protocols employed to improve the management of cancer patients. The journal also focuses on the impact of management programs and new therapeutic

Submit your manuscript here: https://www.dovepress.com/oncotargets-and-therapy-journal agents and protocols on patient perspectives such as quality of life, adherence and satisfaction. The manuscript management system is completely online and includes a very quick and fair peer-review system, which is all easy to use. Visit http://www.dovepress.com/ testimonials.php to read real quotes from published authors. 